\title{
Diversity analysis of paenibacillus cellulase
}

\author{
Wenxi Yue ${ }^{\mathrm{b}}$, Ming Sui*a,b \\ ${ }^{a}$ Key Laboratory of Leather Chemistry and Engineering, Ministry of Education and College of \\ Light Industry, Textile \& Food Engineering, Sichuan University, Chengdu 610065, China \\ ${ }^{\mathrm{b}}$ Department of wine and food engineering,Si Chuan Technology and Business College ,SI \\ chuan,Du jiang-yan 611800,China;
}

Keywords: cellulase; induction; enzyme activity; gel electrophoresis

\begin{abstract}
Cellulose is one of the most abundant biomass resources on the earth. The degradation of cellulose into bio-available sugar is of great significance to the development of bio-energy and bio-based chemicals as well as the comprehensive utilization of biomass resources. A large part of the cellulose degradation method is the use of cellulase degradation. Cellulase includes exo- $\beta$-glucanase, $\beta$-glucosidase, endo- $\beta$-glucanase and the like. In general, cellulase produced by microorganisms in nature belongs to an inducible enzyme, and different substrates may induce a variety of cellulase. In this paper, we focused on the laboratory-screened paenibacillussp.LLZ1, and explored the changes of cellulase activity when the strain was cultured under different carbon sources, revealing the cellulase diversity produced by different carbon sources. The experimental results showed that when using sorbose and cellobiose as carbon sources, cellulase showed the highest activity of enzyme activity and CMC-Naenzyme activity. SDS-PAGE analysis showed that LLZ1 cellulase possessed the natural diversity and could be induced by different substrates Produce different $\beta$-glucanase enzymes.
\end{abstract}

\section{Introduction}

The synthesis of Cellulase is regulated by the strict control of cell metabolism. Understanding the regulation mechanism of cellulase synthesis can effectively change the original regulation mechanism. The biosynthesis of glucanase is mainly regulated by inducers and degradation inhibitors. Because many filamentous fungi can synthesize more than ten-fold of the cellulase substrate in the presence of cellulose, the enzyme synthesis is strongly associated with the culture medium. Studies by Nisiganwa and Canevascmi found that sorbitol, cellobiose, sophorose, lactose, gentiobiose and cellobiose lactones have an induction effect on the production of cellulase, which to varying degrees, increase the synthesis of many cellulase. Cellulase is a class of inducible enzymes. Starch, cellulose, hemicellulose and organic nitrogen are the main components of wheat bran and contain trace amounts of growth factors, which are normally grown and multiplied by their abundant nutrients, while cellulose and hemicellulose may exert their effects on the synthesis of cellulase Has the induction, so that different substrate-induced cellulase activity as well as components and other characteristics are some differences.

Cellulose is a clean, renewable energy source. Now, it has partially replaced fossil fuels. With the shortage and even depletion of fossil fuels, such as oil, and the worsening of environmental pollution, cellulose has received widespread attention because of its large storage capacity, easy source and other characteristics, and its utilization is imperative. At present, cellulase has been successfully applied to the cellulase hydrolysis process due to its specificity and high efficiency. Therefore, how to screen cellulase which has low requirements for the reaction environment, high enzyme activity and good decomposition efficiency has been attracting the attention of domestic and foreign researchers. However, studies have shown that organic solvent and most ionic liquids and cellulase activity inhibition, cellulase in organic dissolution, ionic liquid system instability in situ enzymatic hydrolysis is an urgent need to address the industrial development process. In-depth exploration of different substrate for cellulase induction mechanism can provide a theoretical basis for screening cellulase with high enzyme activity, which is of great significance for solving the 
problem of developing sustainable biofuel and environmental pollution.

\section{Materials and Methods}

\subsection{Experimental Materials}

(A). Paenibacillussp. LLZ1 is a laboratory-selected strain of soil taken from a national forest park.

(B). Sodium carboxymethylcellulose (NaCMC), magnesium sulfate, yeast extract, microcrystalline cellulose (MCC, degree of polymerization 210-240) and Congo red, cellobiose, arabinose, disodium phosphate, dipotassium hydrogenphosphate , Calcium chloride, peptone, sorbose, xylose, agar ammonium nitrate, sucrose, glycerin, galactose and the like.

NACMC plate, slant culture medium (g/L): sodium carboxymethylcellulose 10, peptone 2, magnesium sulfate heptahydrate 0.5 , dipotassium hydrogen nitrate 0.5 , Congo red 0.4 , agar powder 15, yeast extract powder 1 .

Culture medium (g/L): carbon source 5, peptone 1, peptone 1, yeast extract 1, Cacl2 0.5, NH4NO3 0.8, Na2HPO4 • 12H2O 7.56, MgSO4 • 7H2O.

2.2. Experimental methods and procedures

2.2.1. Enzyme activity determination method

(A). Filter paper activity of enzyme activity (FPA) determination:

(1). Method steps: The bacterial liquid was placed in $10 \mathrm{ml}$ centrifuge tube, trimmed, centrifuged centrifuge 4000rpm $5 \mathrm{~min}$, each tube to absorb the supernatant enzyme solution $1 \mathrm{ml}$, placed in a glass tube and added acetate buffer $1 \mathrm{ml}$, water bath at $40{ }^{\circ} \mathrm{C}$ incubated $10 \mathrm{~min}$, Add pre-cut $1 * 3$ filter paper, so that it is completely immersed in the solution, $40^{\circ} \mathrm{C}$ water bath for $1 \mathrm{~h}$ enzymatic reaction, and then remove the filter paper. Add 3mlDNS reagent in the test tube, boiling water bath (5min) in a water bath, and then cooled in an ice bath, measured absorbance at $540 \mathrm{~nm}$ at a moderate dilution; control group after boiling water bath for $10 \mathrm{~min}$, the supernatant enzyme protein After inactivation, the absorbance values were measured after performing the same treatment described above. Then according to the absorbance and glucose standard curve to calculate the glucose content in the measured bacteria, you can compare the total enzyme activity.

(2). Production of glucose standard curve

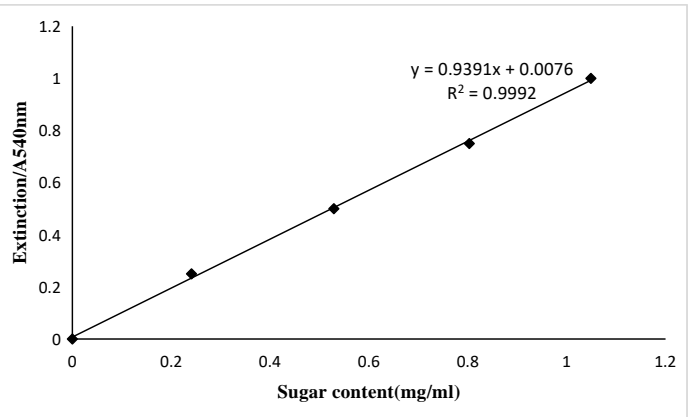

Figure 1. Glucose standard curve

(3). Calculation:

Where $X$ is the activity of the filter paper enzyme enzyme in $U / m L ; W$ is the glucose concentration measured from the glucose standard curve $\mathrm{mg} / \mathrm{ml} ; \mathrm{N}$ is the total enzyme dilution; $\mathrm{T}$ is the reaction time min; $\mathrm{M}$ is the sample volume $\mathrm{ml}$

(B). Determination of enzyme activity by CMC-Na method

(1). Method steps: centrifuge at 4000rpm in a bacterial centrifuge for $5 \mathrm{~min}$, and draw $0.5 \mathrm{ml}$ of the supernatant enzyme solution on the centrifuge tube, add $0.5 \mathrm{ml}$ of $1 \%$ CMC-Na solution and mix thoroughly; water bath $\left(40^{\circ}\right.$ C) for $0.5 \mathrm{~h}$, Immediately after the cold out into the cooling, add $3 \mathrm{mIDNS}$ each tube, water bath boiling water bath $(5 \mathrm{~min})$, and then cooled in an ice bath; the control group is to take $0.5 \mathrm{ml}$ enzyme boiling water bath for $10 \mathrm{~min}$, and then the above operation ; Dilute with water to the appropriate concentration, Absorbance at $540 \mathrm{~nm}$ absorbance measurement. Calculate the glucose content according to the glucose standard curve and calculate the enzyme 
activity.

(2). Calculation:

Where, $\mathrm{A}$ is the dilution factor; $\mathrm{B}$ is the solution glucose content

(C). Determination of total soluble sugar content with sulfuric acid phenol method

(1). Method steps: We will not inactivate that group of bacteria, centrifuged at 4000rpm for $5 \mathrm{~min}$. 4000rpm for the centrifuge speed. Then pipette $0.5 \mathrm{ml}$ of the supernatant. Place the supernatant in a $10 \mathrm{ml}$ centrifuge tube. Then pipette $1 \mathrm{ml}$ acetic acid buffer, into the centrifuge tube, and fully mixed. $40{ }^{\circ} \mathrm{C}$ water bath incubation $10 \mathrm{~min}$. Each centrifuge tube and then add a filter paper, filter paper about $1 * 3 \mathrm{~cm}$ size. The configured centrifuge tube into the water bath at $40{ }^{\circ} \mathrm{C}$ water bath for one hour. After the reaction was completed, remove the filter paper with tweezers. Slowly add 1ml 9\% phenol solution. The reaction solution, which had been added to the phenol solution, was placed in an ice-water bath and $5 \mathrm{ml}$ of concentrated sulfuric acid was slowly added. Shake the tube well. At $485 \mathrm{~nm}$, absorbance was measured.

The deactivation group was centrifuged at $4000 \mathrm{rpm}$ for $5 \mathrm{~min}$. Then you can use a pipette to draw $0.5 \mathrm{ml}, 10 \mathrm{~min}$ has boiled enzyme solution in $10 \mathrm{ml}$ centrifuge tube. Pipette $1 \mathrm{ml}$ of acetic acid buffer solution into the centrifuge tube, fully mixed. $40{ }^{\circ} \mathrm{C}$ water bath incubation $10 \mathrm{~min}$. Each centrifuge tube and then add a filter paper, about $1 \times 3 \mathrm{~cm}$ size. Add water bath at $40{ }^{\circ} \mathrm{C}$ water bath $1 \mathrm{~h}$. After the reaction, remove the filter paper, slowly added $1 \mathrm{ml} 9 \%$ phenol solution. The reaction solution added with the phenol solution was placed in an ice-water bath. Then slowly add $5 \mathrm{ml}$ concentrated sulfuric acid, shake. In normal temperature, wait 30min, color finish. Absorbance was measured at $485 \mathrm{~nm}$. So according to the sucrose standard curve can calculate the total sugar content, did not deactivate the enzymatic reaction with the enzyme activity of the total sugar content of the difference, you can get the enzyme sugar yield, thus calculating cellulaseenzyme activity.

(2). Production of sucrose standard curve:

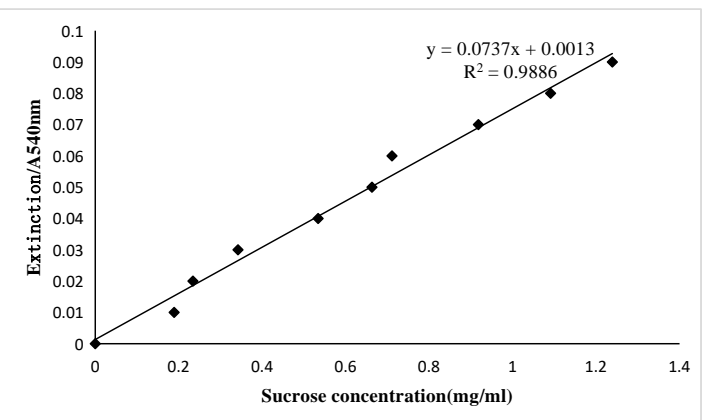

Figure 2. Sucrose standard curve

(3). Calculation:

Where $\mathrm{X}$ is the total sugar content per hundred grams of sample; $\mathrm{C}$ is the sugar content (g) found on the standard curve; $\mathrm{D}$ is the dilution factor $\mathrm{M}$ is the sample weight (g)

2.2.2. Electrophoresis

(A). Preparation of lyophilized enzyme: selected to meet the requirements of carbon source, once again cultured as above, each of the three carbon sources in parallel, cultured, each group to take $500 \mu \mathrm{L}$, mixed, centrifuged at 8000rpm 5min, dispensed to five PCR tubes, Each tube $200 \mu \mathrm{L}$, frozen in a $-80{ }^{\circ} \mathrm{C}$ freezer quickly placed in a vacuum freeze-dryer freeze-dried.

(B). Preparation of plastic plates: cleaning components; Weigh 1\% CMC-Na ultrapure water dissolved and then filtered reserve; Take a bottle of SDS-PEAG electrophoresis solution to dissolve constant volume to 1L; assembly components (glass board alignment, short board inward) Add the lower glue, after injection of ultra-pure water obvious delamination line dewatering, then add the upper glue, insert the comb after half an hour remove the comb backup.

(C). Preparation of different carbon source electrophoresis samples: Select one of the lyophilized enzymes in the culture medium under the suitable carbon source to add $100 \mu \mathrm{L}$ of acetate buffer PH6.0 and mix well. Pipette $8 \mu \mathrm{L}$ of bacteria solution into it, add $6 \mu \mathrm{L}$ of $10 \%$ SDS liquid and 6 $\mu \mathrm{L}$ of non-denaturing non-reducing loading buffer, and then add $10 \mu \mathrm{L}$ of PH6.0 acetate buffer to 
mix evenly; protein molecular weight standard (Marken) before use $40{ }^{\circ} \mathrm{C}$ water bath to melt and draw $10 \mu$ L boiling water bath for $4 \mathrm{~min}$ and then put into cold water for use.

(D). Load sample: Place $10 \mu \mathrm{L}$ marken in the first position; add $20 \mu \mathrm{L}$ sample, $80 \mathrm{~V}$ upper layer and $120 \mathrm{~V}$ lower layer.

(E). Electrophoresis and washing, staining: in turn to each hole by adding the prepared electrophoresis sample; connected to the power supply, at a voltage of $80 \mathrm{~V}$, electrophoresis for 30 minutes, then the voltage of $120 \mathrm{~V}$ electrophoresis 90 minutes; remove the plastic plate, with slow running under water Rinse, the glue washed down. PH6.0 Acetate Buffer 100ml Add 2\% (ie 2ml) Triton and place the gel in a decanter shaker for 40min for the purpose of washing off SDS in the gel; then incubate the gel in acetate buffer at $40{ }^{\circ} \mathrm{C} 2 \mathrm{~h}$, the protein renaturation. After discarding the buffer solution, place the gel in $0.2 \%$ Congo red solution and dye evenly for $30 \mathrm{~min}$. Wash to remove the dye, then $0.85 \%$ saline decolorization about $20 \mathrm{~min}$. Finally, on the orange-red background, there is a clear hydrolyzed band where there is an endo-cellulase activity. Images were taken using a gel imager.

\section{Results and analysis}

\subsection{Cellulase enzyme activity analysis}

\subsubsection{Cellulase filter paper enzyme activity}

This section explored the activity of paenibacillussp.LLZ1 in cellulase induced by different sugars. Filter enzyme activity measured cellulase total enzyme activity. MCC, starch and glycerol because they do not react with the DNS reagent, so use filter paper enzyme activity method (FPA) to measure the cellulaseenzyme activity. $1 \mathrm{ml}$ acetic acid buffer solution was added into $1 \mathrm{ml}$ non-inactivated enzyme solution, the control group was $1 \mathrm{ml}$ boiling water bath $5 \mathrm{~min}$ inactivated enzyme solution was added $1 \mathrm{ml}$ acetic acid buffer solution, and $40{ }^{\circ} \mathrm{C}$ water bath with the same amount of filter paper reaction $1 \mathrm{~h}$, cellulase decomposition filter paper produce glucose . The reaction solution was added with 3mIDNS in boiling water for 5 minutes, rapidly cooled and the reaction solution was diluted to measure the absorbance of the reaction solution, the reaction solution was added with $3 \mathrm{mlDNS}$ in boiling water for 5 minutes, cooled rapidly and the reaction solution was diluted 4 Times, the measured absorbance of the reaction solution using glucose standard curve can calculate the corresponding glucose content, subtraction can be obtained cellulase decomposition filter paper produced by the amount of glucose. Calculated to MCC as a substrate for the production of cellulase can decompose the filter paper yield of $0.145 \mathrm{mg} / \mathrm{ml}$, to MCC sugar yield of $100 \%$, calculated starch and glycerol percentage of sugar content, you can compare the activity of the enzyme size. Calculated, the sugar content of starch was $91 \%$, slightly lower than the MCC, indicating that starch as a substrate induced cellulase activity was relatively high; glycerol content of $12 \%$, indicating that glycerol as substrate-induced cellulase Low activity, glycerol does not well induce cellulase.

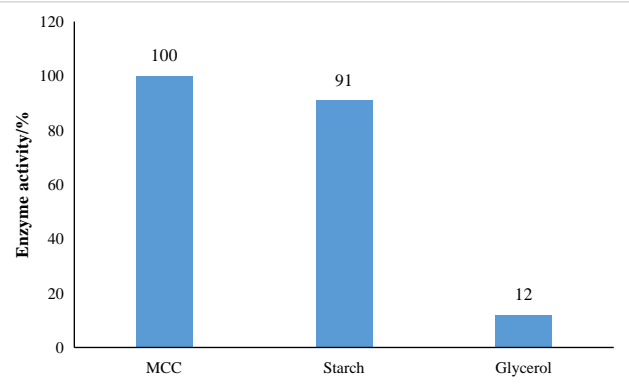

Figure 3. Filter activity of different substrates

Undetectable enzymatic reaction with filter paper decomposes the glucose produced by the cellulose Therefore, the use of sulfuric acid phenol method, the first $1 \mathrm{ml}$ of inactivated enzyme solution was added to $1 \mathrm{ml}$ of acetate buffer, the control group was $1 \mathrm{ml}$ boiling water bath for $5 \mathrm{~min}$ inactivated enzyme solution was added $1 \mathrm{ml}$ of acetate buffer in $40{ }^{\circ} \mathrm{C}$ water bath with the same amount of filter paper Reaction 1h, the decomposition of filter paper to produce glucose, the 
reaction solution was diluted to 40 times, after adding phenol and concentrated sulfuric acid color, measured absorbance of the reaction solution, using sucrose standard curve can calculate the corresponding sugar content, subtraction can be obtained cellulase Decomposition filter sugar production, enzyme activity can be compared to the size. The cellulase produced by MCC was $1.195 \mathrm{mg} / \mathrm{ml}$ and the amount of sugar produced by MCC was $100 \%$, and the content of sorbitol, xylose, arabinose, galactose, Cellobiose, sucrose six kinds of sugar-based substrate for the yield of sugar produced as shown in Figure 4. It can be seen from the bar graph that the yield of sorbose is $92 \%$, which is the highest among the six tested sugars, indicating that sorbose can induce cellulase production in paenibacillussp.LLZ1, and cellulase filter activity high. The sugar content of xylose is $35 \%$, cellulase filter paper activity is lower. The sugar content of arabinose was $42 \%$. Among the six kinds of sugar tested, the activity of filter enzyme was medium but higher than those of xylose, galactose and sucrose. Galactose sugar yield of only 28\%, the lowest filter activity enzyme. Cellobiose sugar yield of up to $91 \%$, and sorbose filter paper enzyme activity or less. The sucrose as a substrate for cellulase activity was $34 \%$, and xylose or less. In general, sorbose and cellobiose filter paper highest activity, and significantly higher than the other several kinds of sugar. In summary, we can see that sorbose and cellobiose are good substrates for inducing cellulase produced by paenibacillusLLZ1. And they produce cellulase decomposition cellulose significantly higher than the ability of several other carbohydrates.

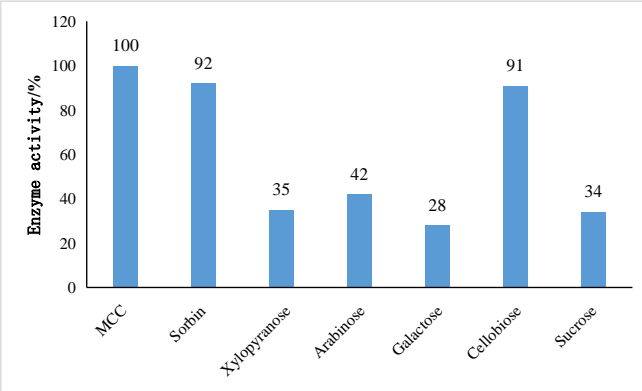

Figure4. Filter activity of different substrates

\subsection{2. cellulaseCMC-Naenzyme activity}

This section explores the CMC-Naenzyme activity of cellulase produced by paenibacillusLLZ1 using different carbon sources as substrate. Because sodium carboxymethyl cellulose is cellulolyzed by endonucleases in cellulase. So it represents the endogenous enzyme activity. The results showed that the endogenous enzyme activity of cellulase produced by MCC was CMC-Naenzyme activity, the amount of glucose decomposed by CMC-Na was $0.072 \mathrm{mg} / \mathrm{ml}$, and the amount of sugar produced was 100\% Figure 5 shows the amount of sugar produced by cellulase produced from starch and glycerol. Endosperm enzyme activity of cellulase produced by starch as a substrate is high, which can reach $85 \%$, indicating that starch can induce cellulase to produce cellulase, and starch is a good inducing substrate. The enzyme activity of cellulose induced by glycerol was only $13 \%$ of that of MCC, and glycerol did not induce cellulase. No significant effect on cellulase activity.

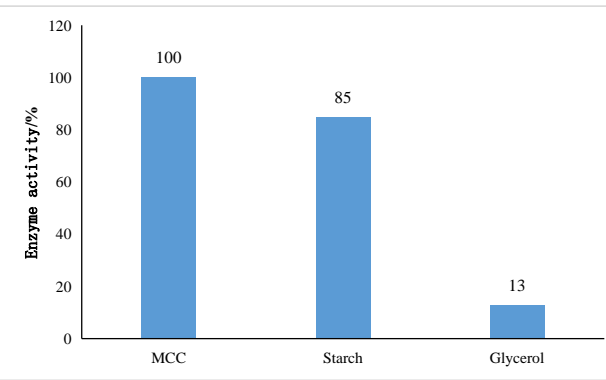

Figure 5. CMC-Na activity of different substrates

\subsection{Electrophoresis results}

After the electrophoresis is complete, the gel is taken out and washed with $2 \%$ Triton in acetate buffer to remove the SDS from the gel. The gel is placed in a $40^{\circ} \mathrm{C}$ water bath in acetate buffer to renature the protein for 2 hours. $2 \%$ congo red staining $30 \mathrm{~min}$, using $0.85 \%$ saline decolorization to 
satisfactory. Finally, a transparent hydrolysing band forms on the red background, with an endo-cellulase activity.

From electrophoresis gel imaging can be seen, to MCC, xylose, galactose substrate for cellulase produced significant electrophoretic bands. Cellulase with MCC as a substrate can see six bands, galactose can see two bands, xylose can see three bands, cellobiose can see three bands, and glycerol can see 2 bands, sucrose can see 3 bands. The results of electrophoresis showed that LLZ1 cellulase has a natural diversity. Different substrate induces different $\beta$-glucanase enzymes.

\section{Summary}

Cellulose is one of the most abundant renewable resources in the world. If cellulose is efficiently used, converting it into bioenergy has far-reaching significance for the sustainable development of the earth. In this paper, the high cellulase enzyme activity strain paenibacillus sp. LLZ1 screened from the national forest park pattern was used to study the diversity of cellulase produced by it. The conclusions are as follows:

In addition to MCC substrate for the culture of the bacteria were also used starch, glycerol, sorbose, xylose, arabinose, galactose, cellobiose, sucrose eight kinds of sugar as substrate culture bacteria, resulting in starch, Yamanashi Sugar, cellobiose-induced cellulase produced by paenibacillussp.LLZ1 has a high enzyme activity.

\section{Reference}

[1] Liao H, Li S, Wei Z, et al. Insights into high-efficiency lignocellulolytic enzyme production by Penicillium oxalicum GZ-2 induced by a complex substrate[J]. Biotechnology for Biofuels, 2014, 7(1):162.

[2] Turumtay H. Cell wall engineering by heterologous expression of cell wall-degrading enzymes for better conversion of lignocellulosic biomass into biofuels.[J]. BioEnergy Research, 2015, 8(4):1574-1588.

[3] Tang L L, Yu X H, Ding A J, et al. Regional contribution to PM 1 pollution during winter haze in Yangtze River Delta, China[J]. Science of the Total Environment, 2016, 541:161-166.

[4] Wang S H, Hung W T, Chang S C, et al. Transport characteristics of Chinese haze over Northern Taiwan in winter, 2005-2014[J]. Atmospheric Environment, 2016, 126:76-86. 